\title{
¿Cómo ve la gente a la sociedad?: la estructura reticular de la opinión pública sobre conflictos sociales
}

\author{
Karl M. van Meter. LASMAS-IdL ${ }^{1}$
}

\begin{abstract}
Resumen. Durante los últimos veinte años, el centro de investigación francés, Agoramétrie, ha realizado, aproximadamente cada año, encuestas a la opinión pública sobre conflicto social. Con una metodología única que incluye una muestra representativa de población francesa y el discurso de los medios franceses sobre conflicto social, se construye un cuestionario cerrado que se utilizará para recoger datos que se analizarán después mediante análisis de componentes principales entre otros métodos. Los sorprendentes resultados después de veinte anos de investigación en el mismo tema muestran que un pequeño grupo de aproximadamente 40 cuestiones "troncales" aparece en cada muestreo de los media sobre conflictos sociales, independientemente de crisis económicas, nacionales o políticas u otros sucesos contextuales. Estas cuestiones "troncales" tales como "hay demasiados trabajadores inmigrantes", "hay médicos de fiar", "deberían tener las mujeres los mismos derechos que los hombres" "los políticos son corruptos" - aparecen como interrogantes humanos básicos sobre la sociedad, y el uso del método de Agoramétrie en Gran Bretaña, Rusia y Costa Rica refuerza esta sorprendente conclusión. Otro resultado que estas encuestas producen es que las cuestiones "troncales", y las otras 50 a 70 cuestiones sobre conflicto social que constituyen el cuestionario cada año no tienen una estructura arbitraria que cambia de una encuesta a la otra. Las cuestiones "troncales" definen cada año una estructura reticular que se repite cada año con muy pocas modificaciones y que mediante sus vínculos ( $y$ oposiciones) define una estructura general con dos dimensiones fundamentales: en primer lugar, una oposición entre una postura abierta hacia la sociedad y sus problemas (los problemas sociales y conflictos de pueden manejar y solucionar) y una postura cerrada ("estábamos mejor en el pasado"); en segundo lugar, una oposición entre una reacción emotiva y una reacción no emotiva al conflicto social. Cada individuo o grupo social, posee una red de opiniones sobre conflicto social, opiniones que no son arbitrarias sino que están relacionadas unas con las otras de manera específica formando redes coherentes cuyos vínculos muestran una fuerte resistencia a la deformación por eventos externos. En resumen, las redes específicas de opinión sobre conflicto social se pueden asociar con individuos, grupos sociales o generaciones y los vínculos de la red muestran una fuerte resistencia al cambio a lo largo del tiempo. Hay incluso la posibilidad de medir la "resistencia" de estos vínculos reticulares.
\end{abstract}

\footnotetext{
Abstract. For the last twenty years, on a more-or-less annual basis, the French research organization, Agoram,trie, has surveyed French public opinion on social conflicts. With a unique methodology involving the representative sampling of both the French population and French media discourse concerning social conflicts, a closed questionnaire is constructed and used to collect survey data which is then analyzed using principal component analysis, among other methods. The rather surprizing results of twenty years of research on the same topic are that a small set of approximately 40 "trunk" questions appear in each sampling of media coverage of social conflicts, independent of economic crises, national or political crises, or

* Traducción realizada por Claudia Aquilar (Departament d'antropologia social, UAB).

${ }^{1}$ LASMAS-IdL, 59 rue Pouchet, 75005 Paris, France; email: bms@ext.jussieu.fr ; tel/fax 33 (0)1 4051 85 19; web: http://www.ccr.jussieu.fr/bms.
} 
other environing events. These "trunk" questions -- such as "are there too many immigrant workers", "are doctors trust-worthy", "should women have the same rights as men", "are politicians corrupt" -- appear as basic human interrogations concerning society, and the use of the Agoram,trie method in Great Britain, Russia and Costa Rica reinforces this surprizing conclusion. Another result that these surveys produced is that "trunk" questions, and the 50 to 70 other questions concerning social conflict which constitute the questionnaire each year, do not have an arbitrary structure which changes from one survey to the next. The "trunk" questions define each year a fundamental network structure that comes back with little modification each year, and in which ties -- and oppositions -- define an overall structure with two fundamental dimensions: first, an opposition between an ouverture toward society and its problems (social problems and conflicts can be addressed and dealt with) and closure ("we were better off in the past"); second, an opposition between emotive and non-emotive reactions to social conflict. Each individual, each social group, possesses a network of opinions on social conflicts, opinions that are not arbitrary but tied one to another to form specific, coherent networks whose ties show a strong resistant to deformation by external events. In short, specific networks of opinions on social conflict can be associated with individuals, social groups and generations, and the network ties show strong resistance to change over time. There is even the possibility of measuring the "resilience" of these network ties.

\section{INTRODUCCIÓN}

La opinión pública se considera habitualmente un tema difícil de investigar por la variabilidad de los resultados de las encuestas, a pesar de las declaraciones acerca de los márgenes de error esperados. En un estudio clásico de la variabilidad en sondeos y encuestas, Converse y Traugott (1986) advirtieron que las mediciones directas de la opinión pública sobre asuntos nacionales aparecían cada vez de manera más frecuente en los medios de comunicación de masas. A menudo se advertían discrepancias entre diversas encuestas realizadas al mismo tiempo sobre lo que parecían ser los mismos temas. Los autores compararon los resultados de las encuestas realizadas por distintas instituciones y revisaron algunas fuentes de variabilidad y sesgo sistemático en tales encuestas, incluyendo el error muestral, la variabilidad en la composición de la muestra, la variabilidad del encuestador y la variabilidad del cuestionario. Groves (1989) publicó también una rigurosa revisión de la cuestión. Desde entonces se han hecho muchos progresos en la mayoría de estos campos (De Heer, de Leeuw and van der Zouwen, 1999). Los medios para llegar al rigor metodológico necesario de modo que se reduzca significativamente la variabilidad existen pero, como Groves mostró, hay un coste por la reducción de la variabilidad.

\section{"MUESTREO REPRESENTATIVO" PARA LA CONSTRUCCIÓN DE CUESTIONARIOS}

Una de las preocupaciones principales en los estudios o encuestas es la construcción de cuestionarios y a pesar de que ha habido una extensa investigación acerca del formato, el lenguaje y el orden de las preguntas o los "efectos halo" (Schwarz y Sudman, 1996), parece que hay poca investigación orientada a establecer un proceso formal para determinar el contenido del cuestionario de tal manera que permita una "representación" estadísticamente válida del fenómeno social que supuestamente se 
pretende estudiar. En resumen, hay ciertas carencias en lo que a encuestas y sondeos se refiere. Por un lado, hay procedimientos formales para generar una muestra estadísticamente representativa de una nación, una población específica o diversos tipos de grupos sociales. Por otro, a menudo un "panel de expertos" determina de una manera empírica o incluso pragmática, el contenido de los cuestionarios para grandes sondeos nacionales, basándose en su "conocimiento personal" y la experiencia anterior con encuestas similares.

Es en este aspecto concreto dónde la metodología de Agoramétrie difiere de las encuestas tradicionales y ha sido capaz de obtener resultados tan estables que han permitido a las principales instituciones francesas -gubernamentales y no-gubernamentales-- hacer previsiones de futuro a largo plazo basadas en los resultados de tales encuestas. Entre uno y varios meses antes de un estudio sobre conflicto social, un centro especializado de análisis de los medios de comunicación ${ }^{2}$ analiza todos los artículos sobre conflicto social publicados en una extensa muestra representativa de los medios impresos franceses. Para el estudio de 1992 la prensa se analizó entre el 15 de diciembre de 1991 y el 31 de enero de 1992. La muestra representativa de los medios incluyó siete diarios de tirada nacional ( $L e$ monde, Libération, Le Quotidien de Paris, Le Figaro, L'Humanité, La Croix y France-Soir), dos periódicos regionales (Ouest-France y La Voix du Nord), nueve semanarios de tirada nacional ${ }^{3}$ y dos mensuales ${ }^{4}$. Para el estudio de 1997, la prensa se analizó desde el 1 de julio de 1997 hasta el 10 de octubre de 1997. Esta vez la muestra representativa de medios incluyó sólo seis periódicos (Le quotidien de Paris y L'Humanité fueron sustituidos por Le parisien) aunque incluyó cinco periódicos regionales ${ }^{5}$, diez semanales ${ }^{6}$ y tres mensuales ${ }^{7}$.

Los distintos conjuntos de temas recogidos de conflicto social se consideran una muestra representativa del universo de discurso publico sobre conflicto social en Francia en ese período concreto. Las distintas entradas están reorganizadas en 27 categorías temáticas que han resultado ser útiles y más o menos exhaustivas. Éstas son: Política nacional, política internacional, escándalos, catástrofes naturales, economía, seguridad, justicia, familia, trabajo, salud-medicina, urbanización-vivienda, fuerzas armadas, energía, educación, religión, agricultura, medio ambienteecología, transporte, problemas morales-sociales, ciencia, informaciónmedia, tercera edad, consumo-alimentación, industria cultural, deporte, ocio, belleza-moda. Dentro de cada una de las 27 categorías, las entradas individuales se agrupan dependiendo del contenido para crear subtemas 0 , a veces, subsubtemas, dependiendo del número y contenido de los registros muestreados.

\footnotetext{
${ }^{2}$ Hedera (70 bis, Rue de Romainville, 75019 Paris; hedera@practique.fr) realizó este análisis para las encuestas entre 1991 hasta la reciente encuesta de 1999.

${ }^{3}$ L'Express, Le Nouvel Observateur, Le Point, L'Evénement du jeudi, Le Figaro Magazine, Elle, La Vie, V.S.D y Paris Match,

${ }^{4}$ Actuel y Globe

${ }^{5}$ Ouest France, La Voix du Nord, Midi Libre, La Montagne y Le Républicain Lorrain.

${ }^{6}$ L'Express, Le Nouvel Observateur, Le Point, L'Evénement du jeudi, Le Figaro Magazine, Elle, Paris Match, Madame Figaro, Le Canard Enchaîné y Charlie Hebdo.

${ }^{7}$ Notre Temps, Pour La Science (Edición francesa de Scientific American) y Science et Avenir.
} 
Por ejemplo, en 1997, la categoría, "política nacional" tenía los siguientes subtemas: elecciones legislativas, convivencia, nuevo gobierno, políticas generales, presupuesto del 1998, política de empleo, horario laboral, política social, política de inmigración y ciudadanía, partidos políticos y Córcega. El subtema "partidos políticos" estaba organizado en los siguientes subsubtemas: Mayoría parlamentaria (dos entradas), partido socialista (una entrada), Partido Comunista Francés (cuatro entradas), Ecologistas (una entrada), Oposición parlamentaria (cinco entradas), RPR conservadores (una entrada), Frente Nacional (siete entradas). Se puede observar que los temas de conflicto social se sitúan alrededor de la extrema derecha (Frente Nacional), la oposición y los Comunistas.

Utilizando este muestreo temático reorganizado de la producción mediática sobre conflicto social, se establece un amplio panel de expertos observadores de problemas sociales desde diversos ámbitos (a menudo hasta 15 personas) que propone una lista de preguntas que es lo más representativa posible de la muestra temática. Para cada estudio la composición del panel ha sido distinta con $50 \%$ o más nuevos miembros. Agoramétrie utiliza la lista de preguntas sugerida para construir el cuestionario de la encuesta que varía en longitud de 70 a 100 preguntas cerradas sobre conflicto social. Normalmente las preguntas están escritas a modo de declaración ("dios existe" o "hay demasiados trabajadores inmigrantes") para alentar al encuestado a "posicionarse" o "situarse". Las categorías de la escala de cinco puntos de respuesta son: "Estoy en total desacuerdo", "no estoy muy de acuerdo" "quizás estoy de acuerdo" "estoy de acuerdo" "completamente de acuerdo". Traducciones de "Pas du tout d'accord", "Pas tellement d'accord", "Peut-être d'accord", "Bien d'accord", "Entièrement d'accord". Hay también una categoría de "no contesta".

\section{LA METOdología de enCUESTAS DE AgORAMÉtRIE A NIVEL NACIONAL E INTERNACIONAL}

El cuestionario construido de esta manera por Agoramétrie se administra a una muestra representativa de población francesa de, normalmente entre 1000 y 2000 personas. Lo administra una importante institución francesa de encuestas $^{8}$. Los datos se analizan mediante un tipo de análisis de correspondencias llamado análisis de componentes principales que es esencialmente una factorización de eigen values de la matriz de datos (personas por respuestas) para obtener los principales ejes. El principal eje ha designado sistemáticamente "compromiso" o "participación en la encuesta", en el sentido de que el encuestado ha evitado el uso masivo de las categorías "no contesta" o "quizás". En lo que queda de articulo no tendremos en cuante este eje al hablar del "primer" el "segundo" y el tercer" eje.

La mayoría de lo que hemos comentado anteriormente no es conocido en la sociología anglosajona por la sencilla razón de que este trabajo se ha llevado a cabo casi exclusivamente en Francia, y ha sido publicado $y$ distribuido directamente por Agoramétrie a una pequeña audiencia especializada. En 1986 el Bulletin de Méthodologie Sociologique (BMS)

\footnotetext{
${ }^{8}$ De 1977 a 1985, SECED, en 1986, SEDEF, y de 1987 a la actualidad, BVA.
} 
publicó (Van Meter, 1986) un análisis (en francés) de Les structures de l'opinion en 1985 (Agoramétrie, 1986), y, en,1987, un artículo (en Francés), "Les structures de l'opinion: Enquêtes et méthodologie"(Las estructuras de la opinión: encuestas y metodología; Agoramétrie, 1987), basado en los dos capítulos metodológicos de Agoramétrie ( 1986). En 1988 se defendió una tesis doctoral en base a la investigación de Agoramétrie y presentó la metodología de encuesta de Agoramétrie detalladamente (Iliakopoulos, 1988). Una de las pocas publicaciones en inglés sobre la investigación de Agoramétrie apareció en el International Journal of Public Opinion Research (Durand et al., 1990).

Sin embargo, esta falta de interés en las corrientes mayoritarias de la investigación sociológica americana no ha impedido que el método se aplicara en otras partes. Vladimir O. Roukavichnikov, el ex-subdirector del Instituto de investigaciones socio-políticas de la academia de ciencias rusa y vice-editor de la revista, Sotziologicheskie Issledovanija, utilizó el método en encuestas de opinión en 1991 y 1992 en Rusia y presentó los resultados en el simposio "Current Developments in Environmental Sociology" en Woudshoth, Holanda, en Junio de 1992 (Rukavishnikov, 1992). Él afirma específicamente que: "El análisis VARIMAX (Sherin, 1966) muestra una notable estabilidad de las estructuras de opinión pública... El primer componente principal corresponde a la dimensión que opone una visión tradicional-conservadora con una moderna-radical... el segundo componente principal --"material" -- parece caracterizar la dimensión que opone frustración con satisfacción..." (ibid: p.7). Esos son exactamente los dos primeros ejes que se encontraron en las encuestas francesas.

Rukavishnikov señaló claramente: "Para nosotros, fue un extraordinario hallazgo que incluso las etiquetas de los ejes en el estudio de nuestros colegas franceses fuesen las mismas que en el nuestro. Pero trabajamos de manera independiente" (ibid).

Los resultados también "mostraron un alto grado de similitud" con los del estudio de Radio Free Europe-Radio Liberty (1990). Se debería remarcar que los resultados de la encuesta rusa se obtuvieron con una serie de 38 preguntas que son bastante diferentes de las de nuestras encuestas francesas.

La utilización de la metodología de Agoramétrie por Rukavishnikov no es el único caso en Europa del Este. Rasa Alisauskiene, ex - director de Baltic Surveys Ltd. En Viniius, Lituania, le comunicó al autor que ella también había utilizado tal metodología. El "cuestionario de un estudio sociológico sobre la opinión pública acerca de los riesgos medioambientales"construido en 1992 por el instituto de sociología de la academia de ciencias Búlgara, consistió en 65 preguntas ampliamente inspiradas por la investigación de Agoramétrie.

En Europa Occidental, el enfoque de Agoramétrie fue utilizado por una organización de encuestas británica, el Instituto Mori, para su estudio Living in Britain 1989. Estos, al igual que los rusos, encontraron los mismos dos ejes en la estructura de la opinión pública sobre conflictos sociales. De nuevo, en este caso, las preguntas generadas por el método de Agoramétrie 
hacían referencia al discurso de los medios británicos sobre conflicto social y no eran una copia directa de las preguntas del estudio francés.

Fuera del "primer" y el "segundo" mundo, la metodología se ha utilizado en Costa Rica en la Escuela de Sociología de la Universidad de San José en investigaciones sobre la opinión acerca de conflictos sociales y, una vez más, se han encontrado resultados similares (Poltronieri, 1999).

\section{los Resultados de la encuesta a lo laRgo de Veinte AÑOS}

Cuáles son exactamente estos resultados encontrados en Francia, Rusia, Gran Bretaña y Costa Rica? Empezando en 1977, en Francia, cada encuesta de Agoramétrie produjo un típico grafico bidimensional cuyos ejes son los dos factores principales de la descomposición eigen-value de la matriz de datos.

Ver el grafico "el cielo de diciembre 1997". En la esquina superior derecha (primer cuadrante), se puede ver "Sentimiento de inseguridad" ("Sentiment d'insecurité"), "Restablecer la pena de muerte" ("Rétablir la peine de mort") y "Demasiados trabajadores inmigrantes" ("Trop de travailleurs immigrés"). Veremos más adelante que éstas tres respuestas no solo caracterizan el área superior derecha del gráfico sino que también forman una de las redes de opinión más fuertes y estables. El lector o la lectora puede imaginar fácilmente qué clase de persona sostendría esta red de opinión particular y qué otros lazos positivos o negativos podrían existir con otras opiniones. 


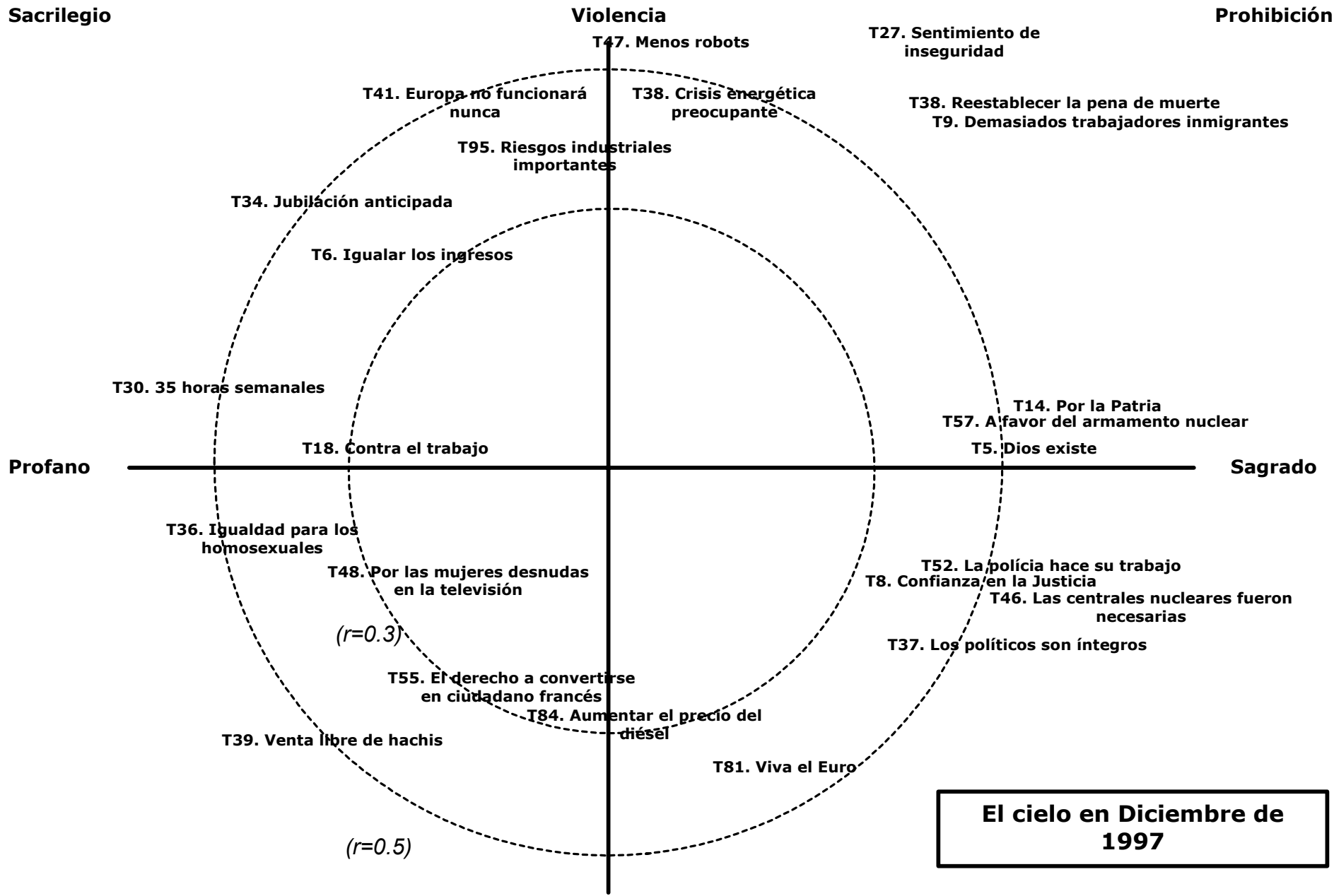

Transgresión

Mesura

Ritual 
En la esquina inferior izquierda, en el tercer cuadrante, se puede encontrar "Venta libre de Hachís" ("Hashish en vente libre"), "El derecho a convertirse en ciudadano francés" ( "Pouvoir devenir Français") y "A favor de la mujeres desnudas en la TV" ("Pour des femmes nues à la télé".) De nuevo aquí, éstos tres ítems caracterizan un área del gráfico, pero están mucho menos ligados entre ellos de manera fuerte y estable formando una red de opiniones comparados con los tres ítems precedentes (con los que se encuentran en fuerte oposición [correlación negativa]).

En la esquina superior izquierda, en el segundo cuadrante, se puede encontrar "Jubilación anticipada" ("Retraite plus jeune"), "Igualar los ingresos" ("Egaliser les revenus") y "Mayor número de riesgos industriales" ("Risques industriels importants"). Estos ítems se encuentran también relativamente poco asociados entre sí y con su área del gráfico.

En la esquina inferior derecha, en el cuarto cuadrante, se pueden ver, agrupados, "Las centrales nucleares fueron necesarias" ("Il fallait des centrales nucléaires"), "Confianza en la justicia" (Confiance en la Justice"), "Los políticos son personas integras" ("Hommes Politiques integres"), "La policía hace su trabajo" ("La Police remplit sa misión") proporcionando una clara caracterización de éste área del gráfico, sin formar una red de opiniones fuerte y estable entre éstos ítems.

En el límite derecho del primer eje horizontal, se encuentra "Por la patria" ("Pour la patrie"), "Dios existe" ("Dieu existe") y "A favor del armamento nuclear [en mi país]" ("Pour la force de disuasión"), caracterizando una actitud claramente conservadora hacia la sociedad y el conflicto social. En el límite opuesto, el izquierdo del primer eje, se encuentra "A favor de las 35 horas semanales de trabajo" ("pour les 35 heures"), "Contra el trabajo" ("Contre le travail") y "Igualdad para los Homosexueles" ("Homosexuels comme les autres"), caracterizando un claro rechazo de las actitudes sociales dominantes.

En el segundo eje, o eje vertical, encontramos, en la parte superior, "menos robots" ("Moins de robots"), "Preocupación por la crisis energética" ("Crise de l'énergie préoccupante"), "Europa nunca funcionará" ("I’Europe ne marchera jamais"), y, en la parte inferior, "Viva el euro" ("Vive I'Euro"), "incrementar las tasas del diesel" ("Augmenter le diesel"), "Construir Europa con el Este" ("Construire I'Europe avec I'Est"), ambas carecen de coherencia temática aparente pero muestran coherencia emocional con "cooperación" o una respuesta no-emotiva hacia la parte inferior y con "conflicto" o una repuesta emotiva hacia la parte superior.

Estas mismas caracterizaciones del primer y el segundo ejes, y los cuatro cuadrantes construidos en consecuencia, se encontraron en todas las, más o menos anuales, encuestas de Francia $^{9}$ y en los otros estudios mencionados anteriormente. Si se comparan los gráficos bidimensionales de las encuestas rusa, británica y francesa se advierte la similitud en las

\footnotetext{
${ }^{9}$ Hubo una encuesta cada año entre el 1977 y el 1999, con la excepción de 1979, 1980 y 1990. Encuestas de mayor abasto tuvieron lugar cada cinco años: 1977, 1982, 1987, 1992 y 1997.
} 
estructuras tanto de los ejes como a menudo de los mismos ítems -- o tipos de temas.

De este modo podemos describir una estructura general de cómo la sociedad ve el conflicto social afirmando que el factor más importante es una oposición entre una adhesión a las actitudes conservadoras dominantes con una cierta "postura cerrada" hacia los conflictos sociales, y diferentes actitudes no-conservadoras con una cierta "postura abierta" hacia el conflicto social. El segundo factor más importante sería una oposición entre una actitud cooperativa no-emocional hacia el conflicto social y una actitud emocional conflictiva. Los cuadrantes, entonces, se caracterizan como postura cerrada conflictiva (primer cuadrante), postura abierta conflictiva (segundo), postura abierta cooperativa (tercero) y postura cerrada cooperativa (cuarto).

Queremos insistir, una vez más, que esta estructura general muy estable se ha encontrado en varios países bastante diferentes entre ellos y en un periodo de tiempo relativamente largo en Francia, veinte años. Así, podemos afirmar con un alto grado de confianza que estos resultados son independientes del contexto económico, político y social y incluso del contexto internacional incluyendo algunos cambios monumentales como la caída del bloque comunista, el fin de la guerra fría, las guerras en el golfo pérsico y en los Balcanes. Esto plantea la cuestión de cuáles son los límites de aplicación de estos resultados. A cuáles sociedades humanas no son aplicables estos resultados? A qué escala son aplicables: sólo a nivel nacional, regional, ciudades, grupos sociales, familias o incluso individuos? Estas cuestiones serán tratadas más adelante después de examinar algunos de los resultados sorprendentemente estables de esta encuesta.

\section{PREGUNTAS "TRONCALES" SOBRE CONFLICTO SOCIAL}

De la descripción anterior el lector o lectora puede comprender fácilmente que el proceso de muestreo de los medios implica que los temas o tópicos sólo recurren sistemáticamente si realmente representan un aspecto esencial del debate público y no intereses particulares de una institución social o incluso de los mismos medios de comunicación. La gran variabilidad del contexto a lo largo de los últimos veinte años en Francia concede un importante peso a esta afirmación.

Que ha revelado este proceso de muestreo sobre conflicto social después de 20 años de generar cuestionarios de 70 a 100 ítems? La encuesta de 1997 proporciona una lista completa de las 351 preguntas que han formado parte de las 19 encuestas de Agorametrie desde 1977 (Agoramétrie, 1998: 4366). Hay un cierto número de temas-cuestiones recurrentes. Hay 8 cuestiones que fueron "muestreadas" cada vez: liberalizar el aborto, construir plantas de energía nuclear, dios existe, igualar los ingresos, confianza en la justicia (en varias formulaciones), reducir el gasto de defensa (y cuestiones similares), el gobierno es inefectivo( y la formulación "los políticos son personas íntegras") y un sentimiento de inseguridad (y variaciones asociadas). Tres temas más aparecieron en 18 de las 19 encuestas: demasiados trabajadores inmigrantes, contra el trabajo, preocupación por la crisis energética. También hay tres temas más que 
aparecieron en 17 de las 19 encuestas: abolir / restablecer la pena de muerte, la TV nos considera a todos estúpidos, censurar ciertos libros.

La lista completa de temas-preguntas que han aparecido en varios cuestionarios se puede reducir o concentrar reagrupando varias formulaciones o preguntas similares hasta obtener una lista de 42 temas, empezando por los mencionados anteriormente. Cuantos de ellos podrían ser considerados estrictamente temas "Franceses" que no se "muestrearían" en el extranjero? Quizás la "semana de 35 horas", pero está ligada a otros temas de trabajo y de todas maneras será probablemente un tema de debate en el mundo desarrollado en un futuro próximo. Resumiendo, la lista de 42 temas no se limita solamente a los conflictos sociales franceses. Esta limitada entonces a los conflictos sociales del mundo desarrollado? De hecho, las cuestiones que hacen referencia a "armas nucleares", "crisis energética", "ecologistas" y "Ordenadores" o "robots" parece que limiten el campo de aplicación a los países más desarrollados. Pero cada una de estas preguntas de países-más -desarrollados parece estar ligada a temas similares y más universales: armas nucleares con gasto en defensa y sentimiento de inseguridad; ecologistas con la destrucción del medio, y ordenadores-robots con el miedo a las nuevas tecnologías. Quedaría la posibilidad de que éstas preguntas estuviesen asociadas específicamente con culturas "cristianas" y deberían llevarse a cabo encuestas similares en Irán, India y Japón, por ejemplo, para examinar tal posibilidad.

Denominamos a éste grupo de 42 temas recurrentes las cuestiones troncales"("questions souches" en francés) sobre conflicto social no sólo porque aparecen sistemáticamente a lo largo del tiempo y en diferentes sociedades, sino también porque determinan en gran parte la estructura general descrita por los dos ejes principales. Esta doble determinación de las cuestiones troncales se ve reforzada por otros resultados estadísticos del análisis de los datos de la encuesta. La distribución de los valores del ítem "no contesta" abarca desde "la Boy's Band está acabada (pasada de moda"the Boy's Band is 'out") (15.3\%), "más poder para el parlamento" $(10.6 \%)$ y "me gusta Ladi $\mathrm{Di}^{\prime}(0.4 \%)$, hasta "los médicos son fiables" $(0.5 \%)$ y "sentimiento de inseguridad" $(0.4 \%)$. El rango de la media (56.83) y el valor de la media "no contesta" (3.09\%) para cuestiones troncales no son significativo por si mismos, pero lo son cuando se los compara con el rango de la media (45.17) y el valor de la media "no contesta" $(4.42 \%)$ para cuestiones "no-troncales".

Junto con algunos "temas calientes" actuales -LadiDi, Algeria, la caza-las cuestiones troncales muestran la máxima correlación con el grupo de variables descriptivas incluyendo localidad, sexo, edad, estudios, religión, categoría profesional-social, tendencia política, partido político (Agoramétrie, 1998: 69-70). La rotación Procrusteana (shönemann, 1996; Mulaik, 1972) para inscribir el análisis de componentes principales de 1997 sobre el diagrama de 1992, los 47 temas comunes en 1992 y 1997 incluyen todas las cuestiones troncales excepto tres (estudiantes, mujeres y items escolares). De las 47 variables, las 31 más estables, que fueron utilizadas como variables activas para superposición, eran todas cuestiones troncales. Las siguientes variables más estables se proyectaron sobre el gráfico resultante para controlar la estabilidad de la rotación Procrusteana. De las 
diez, todas fueron cuestiones troncales 0 variables fuertemente relacionadas, excepto algunos asuntos "actuales": "hacer un esfuerzo para los juegos olímpicos", "hacer algunos sacrificios por Europa".

De las 31 variables que reconstruyen mejor el diagrama del análisis de componentes principales de 1997, sobre la base del análisis de correlaciones múltiples de coeficientes de regresión (Agoramétrie, 1998: 91), todas excepto dos - "En Francia se ha ocultado la verdad sobre Chernobyl", "construir Europa con el Este" - son cuestiones troncales, aunque la primera de estas dos se puede asociar (negativamente) con "los políticos son personas íntegras" y la segunda con "Ayudar a los países subdesarrollados". Finalmente, las cuestiones troncales también figuran entre los grupos de variables que más fuertemente correlacionan con los factores de la matriz de datos, según el análisis VARIMAX.

En resumen, las cuestiones troncales proporcionan un mapa topográfico básico en el que la sociedad proyecta cómo ve el conflicto social. Los puntos de referencia o cuestiones troncales principales se conocen y cambian de posición o amplitud muy lentamente. Es el terreno "actual" o inmediato que puede cambiar mucho más rápido. Pero aunque este resultado parezca bastante bien establecido, no responde a la pregunta de "porqué" o cuán ampliamente aplicable es este resultado a las sociedades humanas. Intentaremos responder a estas cuestiones más adelante.

\section{ESTRUCTURA DE LA RED DE OPINIÓN SOBRE CONFLICTO SOCIAL}

\section{Análisis de redes a nivel poblacional}

Veamos las correlaciones resultantes del análisis VARIMAX (ten Berge, 1984). El primer factor correlaciona, en orden decreciente, con: demasiados trabajadores inmigrantes, en contra de que los extranjeros residentes por un período largo se conviertan en ciudadanos franceses, en contra de ayudar a los países subdesarrollados, a favor de la intervención en Argelia, a favor de la pena de muerte, en contra de dar papeles de residencia a los inmigrantes ilegales, en contra de construir Europa con el Este, a favor de más controles policiales en la calle, sentimiento de inseguridad, Europa no funcionará, el gobierno no es efectivo, ningún sacrificio por Europa. Ésta es una descripción clara y coherente de "postura cerrada" hacia el conflicto social y representa el área superior derecha de nuestro gráfico. No obstante, se debería tener en cuenta que sólo la mitad de los ítems son cuestiones troncales. Aunque todas las variables-temas del grupo correlacionan con el primer factor, ello no implica necesariamente que están ligadas entre ellas, como veremos mas adelante. Aún así, forman una red coherente de temas asociados por su contenido y actitud hacia la sociedad y el conflicto social.

El segundo factor correlaciona con: contra la pornografía, contra las mujeres desnudas en la TV, a favor de censurar ciertos libros y en contra de la venta libre de hashish. El tercer factor: menos robots, la polución destruirá al hombre, los ciudadanos se deberían organizar contra el crimen, 
los ordenadores nos quitan la libertad, en contra de las centrales nucleares, preocupación por la crisis energética y el progreso científico es malo. El cuarto factor: nuestra comida no es natural, la verdad sobre Chernobyl ocultada, las industrias representan un riesgo para nosotros, la TV nos toma por idiotas y demasiada influencia política en el sistema legal. El quinto factor: la policía hace su trabajo, los políticos son personas íntegras, confianza en el sistema legal y confianza en los periodistas. Podemos ver que con factores más altos la coherencia temática tiende a decrecer, pero, hasta ahora, cada grupo ha incluido por lo menos una cuestión troncal y a menudo varias. No será siempre así en adelante. En el sexto factor tenemos esfuerzo patriótico; en el séptimo, pro-negocio/anti-gobierno; en el octavo, contra el trabajo; en el noveno, a favor de la homosexualidad y el aborto; 100, anti-coches; $11^{\circ}$, anti-media; $12^{\circ}$, anti-médicos; $13^{\circ}$, activismo social; $14^{\circ}$, ultra-liberalismo; $15^{\circ}$, control del peso; $16^{\circ}$, a favor de los valores familiares; 170 , igualdad social; $18^{\circ}$, ejército profesional; 190, bienestar y matrimonio y $20^{\circ}$, Dios existe y utilidad del programa de empleo juvenil. Como se puede ver en el factor 20, cuanto mayor es el número del factor, menor es la coherencia temática entre las variables. Sin embargo, con los factores iniciales, el análisis VARIMAX define estadísticamente la red local en la que se sitúa una opinión.

Una descripción más precisa de los vínculos o asociaciones entre opiniones individuales sobre conflicto social la proporciona la serie completa de tabulaciones cruzadas (Agoramétrie, 1998: 184-282). Volviendo al grupo de temas que correlaciona con el primer factor, encontramos que la correlación entre pares más fuerte es entre "demasiados trabajadores inmigrantes" (cuestión troncal) y "restablecer la pena de muerte" (cuestión troncal), seguida de "demasiados trabajadores inmigrantes" (ibid) y (negativa) "los residentes extranjeros por un período largo deberían obtener la ciudadanía" (no es una cuestión troncal). Otras correlaciones fuertes se dan con "no intervenir en Argelia" (no troncal), "castigar a los delincuentes juveniles" (no troncal), "controles policiales en la calle" (no troncal), "gobierno inefectivo" (troncal) y "papeles de residencia para los inmigrantes ilegales" (no troncal). Esta es una actitud extremadamente xenofóbica y represiva que va más allá de la poco precisa red de opiniones correlacionadas con el primer factor y "desarrolla" el presente del las cuestiones troncales con los temas más "actuales" (Argelia y los trabajadores inmigrantes).

En orden decreciente, las otras redes de opiniones que tienen una mayor correlación entre pares son sucesivamente: "contra la pornografía", "contra las mujeres desnudas en la TV", "censurar ciertos libros y en contra de la libre venta de hashish" (similar al segundo factor); "ayudar a los países subdesarrollados" (negativa), "demasiados trabajadores inmigrantes" y (negativa) "no intervenir en Argelia; gobierno ineficiente, sentimiento de inseguridad y demasiados trabajadores inmigrantes"; las siguientes 7 redes correlacionadas por pares son simplemente permutaciones de la primera. Es como si el término "cerrado" fuese una descripción literal con un significado "abierto", por contrato, a opiniones más débiles pero con muchos más lazos diferentes entre ellas.

De hecho, las redes, aparte de las de la esquina superior derecha, son mucho más débiles y variadas, y la estructura de conjunto esta fuertemente 
influenciada por las correlaciones negativas o oposiciones generadas por el bloque cerrado descrito anteriormente.

\section{Análisis de redes del nivel Individual y Generacional}

Además de éste análisis de redes de las opiniones a nivel poblacional, basado en gran medida en las correlaciones estadísticas positivas y negativas, el problema se puede examinar desde otros dos niveles: el del individuo y el de la "generación". La investigación a nivel del individuo es parte de la extensa investigación sobre redes homófilas y el análisis de si la red de opiniones de ego influye en la elección de amistades de ego o si la red de amigos de ego influye en las opiniones de ego. En un estudio clásico, Van der Rijt (1979) observó éste problema con respecto a la juventud y la difusión de información antitabaco, y trabajos recientes han examinado el consumo de drogas y el SIDA.

Leenders (1997) ha proporcionado una visión crítica de la cuestión a nivel individual, señalando claramente que "las causas y las consecuencias de las estructura de la red y los atributos del actor están interrelacionadas" (ibid: 171). A este nivel, según Leenders, "cuando los actores cambian sus patrones de amistad y por consiguiente revisan sus actitudes, lo primero se debe selección, lo segundo a contagio. Alternativamente, cuando los actores cambian sus opiniones lo que lleva a elegir nuevos patrones, lo primero es contagio y lo segundo selección. Esta noción es importante pues muestra que aunque contagio y selección interactúan, son procesos distintos con distintas consecuencias empíricas (cambios en opiniones versus cambios en la estructura de la red)" (item). Aunque sería posible desenredar estas dos causas "interrelacionadas" a nivel individual, el hecho de que ego haya nacido en una generación sin haberlo elegido, con toda probabilidad, establece sus opiniones entre los 15 y los 25 años (Pages et al, 1997), necesariamente significa que el contagio y la selección se darán en un marco determinado bastante diferente del examinado sólo a nivel individual. Sin embargo, la investigación de redes a éste nivel contribuye en gran medida a entender los mecanismos individuales de opinión-construcción de la propia red y parece ser el camino más prometedor a seguir.

A nivel más general, el cambio de opinión a largo plazo parece que es "lento y constante"(Smith, 1994) incluso para los temas más sensibles como la energía nuclear (Pages et al, 1997). Uno de los mejores análisis conocidos del efecto-generación en el cambio de opinión es el de Ronald Inglehart (1979) y su construcción de una escala de 12 items Materialista/Postmaterialista que también utiliza una batería Rokeach de dieciocho ítems para obtener información de "todas las áreas de interés humano con la mínima duplicación" (idem: 317). De acuerdo con Inglehart, "Una de las ideas centrales que impulsó el proyecto actual de investigación fue una hipótesis de cambio intergeneracional en los valores prioritarios de la sociedad occidental. Ésta hipótesis implica la existencia de diferencias persistentes en los objetivos básicos de diferentes generaciones" (idem: 305-306). Inglehart encontró que "las diferencias sustanciales de hecho existen entre los valores prioritarios de las generaciones de ancianos y de jóvenes de los países occidentales. Un grupo de prioridades "Materialistas" (como nosotros las denominamos) tiende a prevalecer entre los 
entrevistados mayores, mientras que las de tipo "postmaterialista" se generalizan de manera creciente mientras vamos analizando progresivamente los grupos más jóvenes" (item: 309). No obstante, en la construcción de su escala Inglehart no explica porqué hay sólo dos categorías (y el eventualmente añade categorías "Mixtas"), porqué éstas categorías deben ser "Materialista" y "Postmaterialista" (caracterizado por su elección de ítems), y cómo se podrían encontrar, definir y construir las categorías futuras.

Aparte de esta crítica de la naturaleza a priori de la construcción de la escala de Inglehart, se ha criticado su aplicación para buscar efectos generacionales en la evolución de la opinión pública sobre la energía nuclear (Pages, et al, , 1997), a pesar de que el equipo de investigación encontró un efecto generacional significativo sobre la red de opiniones acerca de la energía nuclear. De acuerdo con Pages et al (1997: 22), " En resumen, esta lejos de ser evidente que la escala de Inglehart da cuenta del efecto generacional que nosotros encontramos cuando analizamos la opiniones sobre la energía nuclear"

Unos pocos años antes, un equipo de investigadores, usando la metodología de Agoramétrie, encontró efectos generacionales similares en redes generales de opinión pública francesa. En su informe (Durand et al, 1990), el equipo abordó la cuestión en la sección, "Evolución de opiniones en Francia de 1978 a 1988. Identificando los efectos de la fecha, edad y generación" (ibid.: 40-48), dónde identificaron "sujetos estables", figurando, por definición, entre nuestras "cuestiones troncales". Los temas incluidos en "Influencia solamente de la fecha" fueron "preocupación por la crisis energética", "reducir la diferencia salarial", y "los estudiantes son parásitos sociales". Los temas incluidos en "influencia solamente de la edad" fueron "Dios existe", "sacrificar la vida por la patria" y "la familia debe ser la unidad social básica". Los temas incluidos en "influencia solamente de la generación" incluyeron "combatir la pornografía" (51\% en 1978 , y $43 \%$ en 1988) y "censurar ciertos libros" (38\% en 1978 y $32 \%$ en 1988). Los vínculos reticulares entre los dos últimos tópicos son bastante claros. Sin embargo, el estudio concluyó de manera ambivalente en lo que se refiere al efecto de la generación en general en todas las redes de opinión pública: [quizás] "cada generación tiene características específicas y estables, pero quizás el cuestionario de Agoramétrie no es suficientemente adecuado para detectarlas" (ibid.: 47-48). El último estudio del equipo confirmó claramente el efecto de la generación en lo referente a la red específica de temas relacionados con la energía nuclear.

Otro investigador que ha encontrado de manera clara los efectos de la generación en el cambio de redes desde opinión es Tom W. Smith, director del U.S. General Social Survey (Smith, 1994). Según Smith, "1. La mayoría de los cambios de opinión son lentos y continuos. 2. La mayoría de los cambios de opinión son explicables" (ibid.:187)y "El modelo cohorteeducación es la mejor explicación al cambio de opinión. Explica el cambio en función de dos factores inter-conectados: cambio o renovación de cohorte y aumentos de los años de escolarización" (ibid.:188). Smith afirma que esta explicación ha funcionado en los EEUU en referencia a los temas de relaciones raciales, libertades civiles, roles de género, legalización de la 
marihuana y moralidad sexual. De todas maneras nuestro trabajo con Agoramétrie, en Francia, no ha mostrado claramente el rol de la educación, éstos mismos temas figuran entre las "cuestiones troncales" que hemos descrito anteriormente y que juegan un rol dominante en determinar la estructura global de la opinión pública. De hecho, la importancia de los vínculos (positivos o negativos) entre estos temas ya ha sido descrita. Cuando una cohorte de edad establece una nueva configuración de vínculos reticulares entre "cuestiones centrales", esta estructura acompañará a la "generación" de por vida con pequeños cambios menores debidos a la edad (Pages et al. , 1997). La "suma" de estos efectos generacionales constituye la estructura general de la opinión pública que hemos descrito y actúa como un puente entre la construcción de redes de opiniones del nivel individual y del nivel poblacional.

\section{ALGUNAS CONCLUSIONES}

En este punto sería necesario un resumen y algunas aclaraciones. A nivel del gráfico bi-dimensional general resultante del análisis de componentes principales, los dos primeros ejes se definen según las oposiciones postura cerrada-postura abierta y cooperación-conflicto descritas anteriormente. Estas oposiciones, encontradas en las encuestas de Francia, Rusia, Inglaterra y Costa Rica son independientes de cualquier conjunto específico de preguntas. Cada encuesta sobre conflicto social generará una estructura general en la que los ítems apropiados aparecerán en las áreas que hemos descrito. Con el tiempo, diversas series de encuestas generarán los ítems troncales que, sucesivamente, se irán incluyendo en el cuestionario año tras año y en las diversas áreas particulares del gráfico. No se puede afirmar a priori que, por ejemplo, una serie de encuestas sobre conflicto social en Estados Unidos tendrá "demasiados trabajadores inmigrantes" en la esquina superior derecha y siendo una cuestión troncal.

Sin embargo, la estructura general se construye con cuestiones troncales y sus redes que se establecen con un análisis longitudinal como hemos visto anteriormente, pero el contenido específico y la formulación de cada grupo de cuestiones troncales se verá influenciado por el contexto de la encuesta, es decir el momento en el que se lleva a cabo y la sociedad encuestada. El grupo de cuestiones troncales abarcará los mismos temas fundamentales que las cuestiones troncales de la francesa Agoramétrie, y viceversa, pero las cuestiones pueden no ser las mismas. En las encuestas rusas, "sentimiento de inseguridad" estaba asociado fuertemente a "se desmembrará Rusia?" y "tomará el ejército el poder?" mientras que en Francia, inseguridad está vinculada con "demasiados trabajadores inmigrantes" y "restablecer la pena de muerte", las cuales han sido cuestiones troncales en Francia durante los últimos veinte años.

A nivel "local"o el nivel de asociación entre ítems en el diagrama general, hemos definido redes de opiniones en función de la correlación estadística del nivel-poblacional, y los análisis del nivel individual y generacional. Pero estos vínculos son de naturaleza probabilística. Uno no puede tomar a un votante declarado de extrema derecha, que cree que hay demasiados trabajadores inmigrantes en Francia, y afirmar que esta persona estará también a favor de la pena de muerte. No obstante, hay una alta 
probabilidad de que así sea. Estas redes de opiniones a nivel "local" muestran una coherencia temática socialmente reconocida, y etiquetas, tales como " Agitadores izquierdistas", "activistas ecologistas", "fundamentalistas religiosos", "racistas de derechas", "empresarios antigobierno", "patriotas activos" y "tecnócratas", se pueden aplicar fácilmente a muchas redes "locales". Pero estas redes identificables no consisten solo en cuestiones troncales. Como afirmábamos anteriormente, las redes siempre incluyen por lo menos una, y a menudo varias, cuestiones troncales. Esto, sucesivamente, implica que los otros miembros de estas redes (sus opiniones) son susceptibles de cambiar y dependen del momento, la generación y la sociedad estudiada.

A nivel general, las redes locales y las cuestiones troncales, incluidas en las redes locales, constituyen los vínculos y las oposiciones que operan a nivel global, definiendo así redes más y menos amplias y, finalmente, construyendo la estructura global.

\section{INTERROGACIONES HUMANAS BÁSICAS SOBRE LA SOCIEDAD}

\section{Cuestiones de Aplicabilidad en Escala}

El resumen anterior y las aclaraciones sobre las diferencias entre el nivel local y el general, y el rol de las redes de opiniones y de las cuestiones troncales, no responde a las cuestiones que hacen referencia a la escala y el alcance de aplicabilidad de estos resultados. En resumen, dónde se puede aplicar este tipo de análisis esperando estos resultados? En términos de escala, hay pocas dudas de que el análisis de poblaciones nacionales producirá los resultados establecidos, pues tenemos los resultados de Francia, Gran Bretaña, Rusia y Costa Rica. Funcionará a menor escala como, por ejemplo, grupos sociales? Escaladores de los bosques de Fontainbleau cerca de París fueron entrevistados siguiendo la metodología de Agoramétrie y se encontró la misma estructura general de la población francesa (Corneloup, 1993). Un proyecto de tesis actual utilizó la metodología de Agoramétrie para estudiar las actitudes hacia la comida y los productos modificados genéticamente. Se encuestó a 250 personas seleccionadas arbitrariamente entre estudiantes y sus familias y se obtuvo la misma estructura general de la población Francesa (Quillet, 1998).

Estos resultados implican que la estructura general de conflicto social que hemos presentado es "independiente de la escala", se encontraría en cualquier grupo social de cualquier tamaño y sólo dependería del tamaño para ofrecer una replica del diagrama general más completa y detallada. Un corolario a esta afirmación es que la escala de aplicación no tiene límites fijos ni por abajo ni por arriba. De hecho, investigaciones actuales en neuroanatomía funcional, concretamente la del Laboratorio de Neurociencia Afectiva, de la Universidad de Wisconsin en Madison, bajo la dirección de Richard J. Davidson, ha establecido la lateralidad de la actividad de la corteza frontal del cerebro en reacción a imaginería emotiva. En Davidson (1998), el autor investiga el rol de la corteza pre-frontal y la amígdala en las diferencias individuales de reacción emocional (lo que el lama "estilo afectivo") y desordenes afectivos. En Davidson y Irwin (1999), los autores 
revisan las lesiones cerebrales y los estudios de neuro-representación de las emociones y el estilo afectivo, centrándose en el mecanismo normal de emoción. El trabajo de neuro-representación está basado en análisis TEP (tomografía de emisión de positrones) y RMN (resonancia magnética nuclear) (Weng et al., 1999)

Este estudio implica que la lateralidad afectiva derecha esta asociada con reacciones de retraimiento o una postura cerrada frente a imaginería emocional. La lateralidad afectiva izquierda está asociada con tipos de reacciones más abiertas e inquisitivas. La similitud con el primer eje de la estructura general de conflicto social es evidente e implica que los seres humanos pueden tener una capacidad seleccionada evolutivamente, predisposición o propensión que sitúa sus reacciones al conflicto social desarrolladas-individualmente en algún lugar del primer eje que hemos descrito. Que sea éste posicionamiento un proceso consciente o inconsciente, influido por la cultura o la educación, y fijo o modificable en la edad adulta, son cuestiones para posteriores investigaciones en neurociencia y psicología social.

Una consecuencia interesante de la asociación potencial entre la estructura general de conflicto social y la lateralidad de las reacciones a situaciones emocionales es que cada sociedad humana, y cada grupo social de cualquier tamaño, tendrá gente distribuida a lo largo del eje postura cerrada-postura abierta debido a la variabilidad natural. Cualquier decisión de un grupo de personas de prohibir, excluir, apartar, reprimir o eliminar gente de cualquier segmento del primer eje estará luchando en una batalla sin final contra el desarrollo humano. En diversos períodos de su evolución, una sociedad puede necesitar la contribución de personas situadas en diversos segmentos del primer eje. En una guerra o situación de supervivencia, la máxima cerrazón y la mínima apertura pueden ser las mejores estrategias. En un período de calma y plenitud de recursos, la máxima apertura y la mínima cerrazón serían lo mejor. En períodos largos de tiempo, las sociedades o grupos que se han privado de gente asociada con algún segmento del primer eje estarían en una situación de desventaja y con riesgo de desaparecer.

Este mismo argumento se sostiene para el segundo eje de cooperaciónconflicto, pero no hemos encontrado investigaciones similares acerca de su carácter independiente de la escala o su asociación con la actividad cerebral individual. No obstante, si estos dos ejes demuestran ser tan fundamentales para la actividad humana como nosotros creemos, entonces su inclusión en el repertorio de capacidades humanas en algún momento de la evolución debe haber representado una ventaja evolutiva que debería investigarse. Aunque nuestro método de investigación sociológica requiere del uso del lenguaje para la comunicación, con otros métodos -- como observación a largo plazo-se podría ver si los animales, además de los seres humanos, se distribuyen en un eje postura abierta-postura cerrada en lo referente a conflictos sociales o si otras estructuras, como jerarquías rígidas, tratan tales problemas.

\section{Cuestiones de Aplicabilidad en Alcance}


En el extremo superior de la escala de aplicación, parece que no hay un limite fijo impuesto por la escala, pero esto solo puede ser confirmado para encuestas del tipo Agoramétrie en culturas no-cristianas, como hemos mencionado anteriormente. El extremo superior de aplicación es más probable que se defina por nuestros criterios de aplicación que, concretamente, son la posibilidad de construir una muestra representativa de discurso público sobre conflicto social. Es decir, si hay barreras comunicativas - diferentes lenguas, separación geográfica, exclusión social, separación temporal, regímenes represivos - entonces no se puede construir la muestra y la metodología y los resultados descritos anteriormente no se pueden aplicar en la forma en la que han sido presentados. Por ejemplo, una muestra de los medios soviéticos de finales de los 80 sobre temas de conflicto social no habría producido los mismos resultados que el estudio de Roukavichnikov unos años después. Estos criterios de aplicabilidad impondrían un límite superior en la escala de aplicación del método. Sin embargo, las recientes protestas de Seattle, Washington, contra la Organización Mundial de Comercio muestran que el proceso de globalización afecta no sólo al comercio sino la comunicación y el discurso público sobre conflicto social a escala mundial. Por lo tanto, el límite superior en la escala de aplicación se está haciendo cada vez más alto y podría incluir a toda la población mundial en un no tan distante futuro.

El método, como lo hemos presentado aquí, esta basado en coger una muestra de la prensa sobre temas de conflicto social, pero esta, por supuesto, no es la única manera de obtener una muestra representativa del debate público. Se podrían utilizar muestras de otros medios públicos y en los países más desarrollados con un uso extendido de Internet, se podría analizar el discurso de las salas públicas de chat. Para estudios históricos sería posible utilizar muestras de material de archivo, aunque no necesariamente representativas. En resumen, tomar una muestra de la prensa escrita, cuando es posible, en un procedimiento eficaz pero no la única posibilidad.

\section{Roturas en las redes, resistencia y fortaleza}

Otro aspecto del alcance de la aplicabilidad serían condiciones en las que el método no funcionase y fracasase. Aunque eso no ha sucedido todavía, existe la posibilidad de que eventos catastróficos como una guerra civil en un país desarrollado o una guerra mundial pudiese romper o cambiar los vínculos que unen la estructura general del conflicto social. Teniendo en cuenta lo que hemos presentado anteriormente acerca de las redes de opiniones y las generaciones, un cambio de estas características implicaría un cambio generacional más que un cambio de opinión de gripos de adultos, una idea que aparece a menudo en los estudios de cambio social. Que no hayamos observado un cambio tan radical a lo largo de veinte años de encuestas de opinión en Francia es un indicador claro de que los vínculos que sostienen la estructura general resisten el paso del tiempo sin deformarse. No obstante, los vínculos no son rígidos, como hemos podido observar en los lentos pero progresivos cambios que se dan en la estructura. Por lo tanto los vínculos tienen una cierta resistencia a la deformación. La última cuestión que deberíamos preguntarnos aquí es si sería posible medir la resistencia de estos vínculos. 
Debería ser posible construir una medida comparando la importancia de un tema en sucesivas muestras de prensa y su subsiguiente desplazamiento en la estructura general. Se podía utilizar el número de artículos, el espacio que ocupan en centímetros cuadrados y durante cuanto tiempo aparecen, para construir una medida compuesta de estas tres cantidades y compararla con cuánto se mueve el ítem de una encuesta a la otra en el diagrama general. Esto proporcionaría una medida de resistencia y sucesivamente una distribución de las fuerzas de resistencia descriptivas para todos los ítems. Aunque no proporcionaría un indicador de la fortaleza de un vinculo particular (a no ser que se observe una ruptura), la distribución de las fuerzas de resistencia probablemente describiría una curva de Gauss y proporcionaría un buen indicador del límite inferior de ruptura.

\section{REFERENCIAS}

Agoramétrie (1986), Les structures de l'opinion en 1985, Agoramétrie, Paris.

Agoramétrie (1987), "Les structures de l'opinion: Enquêtes et méthodologie", Bulletin de Méthodologie Sociologique, n. 14 (April), pp. 4-39.

Agoramétrie (1998), Les structures de l'opinion fin 1997, Agoramétrie, Paris.

J. Corneloup (1993), Escalades et société: Contribution à l'analyse du système, du communicationnel et du social, Doctoral thesis in sports sciences (STAPS), Université Paris-Orsay, 3 volumes, 730 pp.

P. E. Converse and M. W. Traugott (1986), "Assessing the Accuracy of Polls and Surveys", Science, 28 November, pp. 1094-1098.

R. J. Davidson (1998), "Affective Style and Affective Disorders: Perspectives from Affective Neuroscience", Cognition Emotion, 12, pp. 307-330.

R. J. Davidson and W. Irwin (1999), "The Functional Neuroanatomy of Emotion and Affective Style", Trends in Cognitive Science, 3, pp. 1121.

W. de Heer, E. D. de Leeuw and J. van der Zouwen (1999), "Methodological Issues in Survey Research: A Historical Review", Bulletin de Méthodologie Sociologique, n. 64 (October), pp. 25-48.

I. L. Dryden and K. V. Mardia (1998), Statistical Shape Analysis, Wiley, Chichester, especially chapter 5, "General Procrustes Method".

J. Durand, J.-P. Pagès, J. Brenot and M.-H. Barny (1990), "Public Opinion and Conflicts: A Theory and System of Opinion Polls", International Journal of Public Opinion Research, vol. 2, n. 1, pp. 3052.

R. M. Groves (1989), Survey Errors and Survey Costs, Wiley, New York.

A. Iliakopoulos (1988), "Etude de l'opinion et enquêtes de référence. Aspects théoriques, méthodologiques et informatiques", Doctoral 
thesis in scientific methods of management, Université de ParisDauphine.

Ronald Inglehart, 1979, "Value Priorities and Socioeconomic Change", in Samuel H. Barnes and Max Kaase (editors), Political Action: Mass Participation in Five Western Democracies, Sage, Beverly Hills CA, pp. 305-342.

Roger Th. A.J. Leenders, 1997, "Longitudinal Behavior of Network Structure and Actor Attributes: Modeling Interdependence of Contagion and Selection", in Patrick Doreian and Frans N. Stokman (editors), Evolution of Social Networks, Amsterdam, Gordon \& Breach, pp. 165-184.

S. A. Mulaik (1972), The Foundations of Factor Analysis, New York, McGraw-Hill; especially chapter 12, "Procrustean Transformations". Jean-Pierre Pages, Philippe Ansel and Laurent Leger, 1997, Des Systemes de Valeurs aux Opinions sur le Nucleaire, Commissariat a I'Energie Atomique (CEA), Direction de la Communication, Paris.

J. Poltronieri (1999), Evolucion de las estructuras de la opinion publica en Costa Rica 1988-1999: Principales resultados estatisticos de las encustas nacionales de 1988 a 1999, Escuela de Matimaticas, Universidad de Saint Jose, Costa Rica.

V. Quillet (1998), "Perception des risques et délibération publique: Des radiations aux gènes", presented at "Les Troisièmes Entretiens Scientifiques de Brest", Brest, 22-23 October 1999; thesis DESS, Université de Versailles-Saint Quentin.

Radio Free Europe-Radio Liberty (1990), "Media and Communication in the USSR", Radio Free Europe-Radio Liberty, Munich.

V. Rukavishnikov (1992), "Public Opinion Structures and Environmental Concerns in Modern Russia", presentation at the "Current Developments in Environmental Sociology" symposium, Woudshoth, the Netherlands, 17-21 June 1992, polycopy, 21 pp.

N. Schwarz and S. Sudman (1996), Answering Questions: Methodology for Determining Cognitive and Communicative Processes in Survey Research, Jossey-Bass, San Francisco.

R. J. Sherin (1966), "A Matrix Formulation to Kaiser's VARIAMAX Criterion", Psychometrika, 31, pp. 535-538.

P. H. Shönemann (1966), "Generalized Solution of the Orthogonal Procrustes Problem", Psychometrika, 31, 1, pp. 1-10.

Tom W. Smith, 1994, "Is There Real Opinion Change?", International Journal of Public Opinion Research, 6, 2, 187-203.

G. M. F. ten Berge (1984), "Joint Treatment of VARIMAX Rotation and the Problem of Diagonalizing Symmetric Matrices Simultaneously in the Least Squares Sense", Psychometrika, 44, 3 pp. 347-358.

Joseph van der Rijt, 1979, Jeugd en anti-rookinformatie. Een onderzoek naar de invloed van 'peer groups' op roken en op het effect van antirookinformatie bij de jeugd, Universiteit van Nijmegen, Nijmegen NL.

K. M. van Meter (1986), "L'Opinion", Bulletin de Méthodologie Sociologique, n. 12 (October), p. 52, (review of Agoramétrie, Les 
Structures de l'opinion en 1985 (1986, Agoramétrie, Paris).

X. Weng et al. (1999), "Imaging the Functioning Human Brain", Proceedings of the National Academy of Science, 96, 11073. 